\title{
BMJ Open Involvement of people who inject drugs in injection initiation events: a cross- sectional analysis identifying similarities and differences across three North American settings
}

\author{
Charles Marks, ${ }^{1,2,3}$ Stephanie A Meyers, ${ }^{1,2,3}$ Sonia Jain, ${ }^{4}$ Xiaoying Sun, ${ }^{4}$ \\ Kanna Hayashi, ${ }^{5,6}$ Patricia Gonzalez-Zuniga, ${ }^{1}$ Steffanie A Strathdee, ${ }^{1}$ \\ Richard S Garfein, ${ }^{7}$ M J Milloy, ${ }^{6,8}$ Kora DeBeck, ${ }^{6,9}$ Kevin Cummins, ${ }^{10}$ \\ Dan Werb (1D ${ }^{1,11}$
}

To cite: Marks C, Meyers SA, Jain $\mathrm{S}$, et al. Involvement of people who inject drugs in injection initiation events: a cross-sectional analysis identifying similarities and differences across three North American settings. BMJ Open 2021;11:e046957. doi:10.1136/ bmjopen-2020-046957

- Prepublication history for this paper is available online. To view these files, please visit the journal online (http://dx.doi org/10.1136/bmjopen-2020046957).

Received 13 November 2020 Accepted 26 July 2021

Check for updates

(c) Author(s) (or their employer(s)) 2021. Re-use permitted under CC BY-NC. No commercial re-use. See rights and permissions. Published by BMJ.

For numbered affiliations see end of article.

Correspondence to

Dr Dan Werb;

dwerb@health.ucsd.edu

\section{ABSTRACT}

Objectives People who inject drugs (PWID) play an integral role in facilitating the entry of others into injection drug use (IDU). We sought to assess factors influencing PWID in providing IDU initiation assistance across three distinct North American settings and to generate pooled measures of risk.

Design We employed data from three PWID cohort studies participating in PReventing Injecting by Modifying Existing Responses (PRIMER), for this cross-sectional analysis. Setting Tijuana, Mexico; San Diego, USA; Vancouver, Canada.

Participants A total of 2944 participants were included in this study (Tijuana: $n=766$, San Diego: $n=353$, Vancouver: $\mathrm{n}=1825$ ).

Measurements The outcome was defined as recently (ie, past 6 months) assisting in an IDU initiation event. Independent variables of interest were identified from previous PRIMER analyses. Site-specific multiple modified Poisson regressions were fit. Pooled relative risks (pRR) were calculated and heterogeneity across sites was assessed via linear random effects models.

Results Evidence across all three sites indicated that having a history of providing IDU initiation assistance (pRR: $4.83,95 \% \mathrm{Cl}: 3.49$ to 6.66 ) and recently being stopped by law enforcement (pRR: 1.49, 95\% Cl: 1.07 to 2.07) were associated with a higher risk of providing assistance with IDU initiation; while recent opioid agonist treatment (OAT) enrolment (pRR: $0.64,95 \% \mathrm{Cl}: 0.43$ to 0.96 ) and no recent IDU (pRR: $0.21,95 \% \mathrm{Cl}: 0.07$ to 0.64 ) were associated with a lower risk. We identified substantial differences across site in the association of age $\left(1^{2}: 52 \%\right)$, recent housing insecurity $\left(I^{2}: 39 \%\right)$ and recent non-injection heroin use $\left(1^{2}\right.$ : $78 \%)$.

Conclusion We identified common and site-specific factors related to PWID's risk of assisting in IDU initiation events. Individuals reporting a history of assisting IDU initiations, being recently stopped by law enforcement, and recently injecting methamphetamine/speedball were more likely to have recently assisted an IDU initiation. Whereas those who reported not recently engaging in IDU and those

\section{Strengths and limitations of this study}

- This is the first study to assess common and differential risk factors for assisting injection drug use initiation across different geographic sites.

- By applying Zou's modified regression and extracting relative risks instead of 0 Rs, the results may be readily applied to mathematical modelling studies looking at the initiation of injection drug use.

- Due to the cross-sectional nature of this study, our ability to evaluate the causal relationship between identified risk factors and assisting injection initiation is limited.

Due to the small number of sites (three), our ability to quantiatively identify heterogeneity across sites is also limited.

recently enrolled in OAT were less likely to have done so. Interventions and harm reduction strategies aimed at reducing the harms of IDU should incorporate contextspecific approaches to reduce the initiation of IDU.

\section{BACKGROUND}

North America is currently facing an opioid overdose epidemic, causing the United States Department of Health and Human Services to declare a public health emergency in 2017. ${ }^{1}$ As of January 2019, it was estimated that over 130 people died each day the previous year as a result of opioid-related overdose in the USA. ${ }^{1-3}$ Given the increased presence of potent synthetic opioids such as fentanyl and carfentanil in illicit drug markets in North America, people who inject drugs (PWID) are exposed to a greater risk of overdose. There are an estimated 2.6 million PWID in North America, ${ }^{4}$ among whom $45 \%$ (>1 million) have experienced an overdose. ${ }^{5}$ 
This implies that injection drug use (IDU) is a key driver of overdose, and that preventing IDU is key to reducing population-level overdose mortality. PWID who practice unsafe injection drug use (IDU) are also at high risk of HIV and hepatitis B and C transmission, and are especially vulnerable to these infections within the first few years of initiating IDU. ${ }^{46}$ Given the increase in the intensity of these risks in the months immediately after IDU initiation events, as well as the difficulty in preventing IDU-related causes of morbidity and mortality once people begin to inject, experts have suggested that efforts to prevent IDU-related harms should be focused upstream towards preventing IDU initiation. ${ }^{89}$

To that end, a large and growing evidence base has established that PWID play an integral role in the process of IDU initiation, with at least $75 \%$ of PWID across a variety of settings reporting being assisted in their IDU initiation events by another person experienced with drug injecting. ${ }^{10}$ The PReventing Injecting by Modifying Existing Responses (PRIMER) study has identified a range of factors placing PWID at increased likelihood of providing IDU initiation assistance to injection-naïve individuals across differing North American contexts (Vancouver, Canada; Tijuana, Mexico and San Diego, USA). ${ }^{8}$ These include age, ${ }^{11}$ gender, ${ }^{11}$ injection frequency, the use of particular drug types (eg, opioids, crystal methamphetamine), non-injection drug use, ${ }^{12}$ criminal justice system involvement, ${ }^{13}{ }^{14}$ homelessness ${ }^{15}$ and access to opioid agonist treatment (OAT) ${ }^{16-18}$ While these findings reveal important similarities and differences, no effort has yet been made to pool findings across settings to assess heterogeneity in risk factors for IDU initiation assistance provision.

We therefore sought to pool findings to assess the heterogeneity of factors related to assisting IDU initiation in San Diego, Tijuana and Vancouver in order to establish a baseline understanding of common and site-specific factors influencing the process of IDU initiation.

\section{METHODS \\ Setting}

PRIMER is a cohort consortium study seeking to identify factors influencing the provision of IDU initiation assistance among PWID, and to investigate whether interventions to reduce HIV risk among PWID may also be effective in preventing this behaviour. ${ }^{19}$ The methods used in the PRIMER study have been previously described in full. ${ }^{19}$ In brief, PRIMER includes quantitative data collected beginning in August 2014 from existing prospective community-recruited open cohort studies of PWID including the Proyecto El Cuete IV (ECIV) cohort (Tijuana, Mexico), the Study of Tuberculosis, AIDS and Hepatitis C Risk (STAHR II) cohort (San Diego, USA) and the linked Vancouver Drug Users Study (VDUS) and AIDS Care Cohort to evaluate Exposure to Survival Services (ACCESS). All of these cohort studies sought to investigate HIV risk behaviours among PWID living in urban settings, and ECIV and STAHR II were specifically designed as a linked binational study mechanism with highly comparable survey items. ${ }^{20}$ ECIV inclusion criteria were that participants be 18 years or older, report IDU in the prior month, speak Spanish or English, currently be living in Tijuana with no plans to relocate and not be participating in intervention studies. ${ }^{19}$ STAHR II inclusion criteria were that participants be 18 years or older, report IDU in the past month, speak English or Spanish and had no plans to move away in the next 24 months. ${ }^{19}$ For Vancouver, VDUS comprised two merged cohorts, the Vancouver Injection Drug Users Study (VIDUS) and the At-Risk Youth Study (ARYS). Inclusion criteria for the VIDUS cohort were that participants be 18 years or older, report injection drug use in the prior month and be HIV negative. Inclusion criteria for the ARYS cohort were that participants be between the ages of 14 and 26 years at baseline, report illicit drug use in the past month and either have experienced homelessness or accessed services aimed at aiding youth experiencing homelessness in the prior month. The other linked Vancouver-based cohort, ACCESS, included participants 18 years or older at baseline, living with HIV and reporting illicit drug use other than or in addition to cannabis in the prior month. ${ }^{19}$ For the current study, only those who had reported a history of IDU were included for analysis. PRIMER interviews collected data on the involvement of PWID in providing IDU initiation assistance as well as participants' selfreported sociodemographic information, substance use, incarceration history, OAT enrolment and other related factors. Baseline PRIMER data collected between August 2014 and December 2016 from ECIV $(n=766)$, STAHR II $(n=353)$ and VDUS/ACCESS $(n=1825)$ will be the focus for the present study. While longitudinal data were available for ECIV and VDUS/ACCESS, only cross-sectional data were available for STAHR II. As such, to maximise comparability across all three sites, we employed only baseline, cross-sectional data from each site. Since PRIMER involves linking distinct and pre-existing cohort studies, the baseline PRIMER data do not correspond necessarily to baseline cohort data.

\section{Measures}

All data are self-reported and capture 'recent' (defined as within the past 6 months) factors of interest. For this study, the outcome of interest was reporting having recently assisted at least one person with IDU initiation in the prior 6 months, coded dichotomously (yes/no). Independent variables of interest were chosen based on findings from published peer-reviewed PRIMER studies identifying site-specific factors associated with IDU initiation assistance provision. These include the following: age (in years) $)^{1112}$; gender (male/female $\left.{ }^{*}\right)^{11-13}$; years since first $\mathrm{IDU}^{11}$; recent IDU (yes/no) ${ }^{18}$; having ever assisted an IDU initiation prior to the past 6 months (yes/no) ${ }^{18}$; recent housing insecurity (yes/no: defined in Vancouver as recently experiencing homelessness; in San Diego and Tijuana defined as whether or not participants reported 
living in at least one of the following places in the prior 6 months: on the streets, in an abandoned building, at their place of work, in a migrant worker camp, in a vehicle, at a shooting gallery or in a homeless shelter) ${ }^{16}$; having recently been stopped by law enforcement (yes/ no $)^{13}$; having recently been incarcerated (yes/no $)^{21}$; recent enrolment in OAT (yes/no) ${ }^{16-18}$; recent methamphetamine injection (yes/no) ${ }^{16}{ }^{18}$; recent speedball (heroin and cocaine combined) injection (yes/no) ${ }^{16}{ }^{18}$; any recent non-injection use of heroin, cocaine or methamphetamine (yes/no) as well as any non-injection use of heroin, cocaine or methamphetamine (yes/no, for each).$^{12}$ Regarding gender, across all cohorts, participants were asked if they were 'male', 'female' or 'transgender'. However, because of data sharing restrictions, we were unable to access data on all gender categories (ie, other than 'male' / 'female') for the Vancouver cohort. Five individuals in the STAHR cohort and zero in the ECIV cohort identified as 'transgender', meaning we did not receive information about their gender identity. In-line with past research, and due to shared vulnerabilities between the two groups, ${ }^{22}$ we opted to include those that identified as 'female' and as 'transgender' within the same group. We have opted to label this variable 'male/female*'.

\section{Statistical analyses}

For each of the three sites, modified Poisson regression models were fit to assess the relationship between identified variables and recently assisting IDU initiation. Age (by 10-year increment), gender, years since first injection, recent IDU and history of assisting IDU initiation were chosen as control variables across all three sites. These five variables were chosen as controls because they address: (1) demographics; (2) injection drug use behaviours and (3) long-term history of having provided injection drug use assistance. Distinct regression models were fit to assess the relationship between each identified factor and their relationship with providing IDU initiation assistance, controlling for the five noted variables. Models assessing recent methamphetamine IDU and recent speedball IDU did not include recent IDU as a control variable to protect from the effects of confounding.

The modified Poisson regression returns log-relative risk point estimates, which we presented as relative risks. ${ }^{23}$ Given recent publications aimed at predictive modelling of population patterns of IDU initiation, ${ }^{18} 24$ we determined that calculating relative risks (as opposed to using logistic regression to calculate ORs) would provide greater utility to future modelling efforts while still applying appropriate statistical rigour. This is because the modified Poisson regression with robust variance estimation (ie, a 'sandwich' estimator) provides a statistically consistent estimate of relative risk and its estimation variance. ${ }^{23}$ The modified Poisson regression model is preferable to the use of logistic regression where an estimate of relative risk is sought, as logistic regression does not provide an unbiased estimation of relative risk except in the special case of case-control studies. ${ }^{25}$
Once modified Poisson models were fit, a meta-analytic approach using participant data from across all sites was used to assess heterogeneity and to compute pooled relative risks (pRR) for each predictor. This is consistent with the definitions laid out by Blettner et al, ${ }^{26}$ where metaanalysis is used to assess site heterogeneity and compute pRR. This approach was preferable to pooling data from all three sites into a single model because each of the parent studies was designed and implemented independent of each other with separate protocols, which may have led to variations in population sampling and covariate data collection for PRIMER. Specifically, logrelative risks extracted from the modified Poisson regression models were assessed for heterogeneity using a restricted maximum-likelihood estimator and pooled by fitting linear random effects models, applying log-SEs to establish study weight. Higgins $\mathrm{I}^{2}$ was generated to assess site heterogeneity for each variable (excluding those included in a syringe-related risk behaviour subanalysis restricted to data from participants in San Diego and Tijuana, as outlined below). ${ }^{27} \mathrm{I}^{2}$ presents the percentage of estimated variance that can be attributed to site heterogeneity; an $\mathrm{I}^{2}$ of $0 \%$ indicates that the differences across study are explained entirely by sampling error, while an $\mathrm{I}^{2}$ of $100 \%$ indicates that the differences across study are explained entirely by site heterogeneity. All analyses were performed in $\mathrm{R}$, with meta-analysis performed by applying the $r m a$ function in the metafor package. ${ }^{28}$

We present the results stratified by whether a given variable's association with providing IDU initiation assistance was homogenous or heterogenous across site. Due to low power to assess heterogeneity, and to ensure conservative thresholds of hetereogeneity, variables with an $\mathrm{I}^{2}>0 \%$ are presented as heterogenous. We assessed associations as homogeneous if they were in the same direction across all three sites (given the absence of tests to assess homogeneity). For all variables, we present site-specific and pRR along with their respective CIs, $\mathrm{p}$ values and $\mathrm{I}^{2}$ values.

\section{Subanalysis: the association between IDU risk behaviours and IDU initiation assistance across sites}

IDU-related risk behaviours were assessed in San Diego and Tijuana, but not in Vancouver, as a result of limited data access. These behaviours were: recently providing a used syringe to another person to inject with (yes/no); recently injecting with a used syringe (yes/no); recently injecting shared drugs via front-loading or back-loading (ie, when drugs are divvied out between PWID by using one syringe to fill another syringe (yes/no) and recently sharing drug preparation equipment (such as cookers, water or cotton swabs) prior to IDU (yes/no). In addition to these four categorical variables, an IDU-related risk score, ranging from 0 to 4 , was calculated by summing together all positive responses to the four IDU-related risk behaviour variables, in line with previous studies. ${ }^{29}$ The same meta-analytic approach as described above was used to calculate site-specific and pRR, although we do 
not present an assessment of site heterogeneity for the subanalysis.

\section{Results evaluation framework}

Consistent with emerging statistical recommendations in the field calling for an end to reliance on bright-line significance testing, ${ }^{30-32}$ we opt to report study findings by applying the postsignificance communication structure (POCS) ${ }^{33}$ Instead of relying on null hypothesis significance testing to evaluate study findings, through POCS we make an evaluation of point estimates, CIs and corresponding $\mathrm{p}$ values in relation to the underlying scientific questions to make study conclusions. ${ }^{33}$ As such, we consider $\mathrm{p}$ values as continuous rather than dichotomous variables and refrain from denoting significance based on a bright-line value, $\alpha$.

\section{Patient and public involvement}

Neither patients nor the public were involved in the design, conduct, reporting or dissemination plans of our research. The full protocol of the PRIMER study has been described elsewhere. ${ }^{19}$

\section{RESULTS}

\section{Participant characteristics}

Overall, 2944 participants contributed data for this study from Tijuana $(n=766)$, San Diego $(n=353)$ and Vancouver $(\mathrm{n}=1825)$ (table 1). Of these participants, $41(5.4 \%), 18$ $(5.1 \%)$ and $88(4.8 \%)$ reported recently providing IDU initiation assistance, respective to each site (total $n=147$; $4.9 \%$ ). Average age of participants was 40 years in Tijuana (IQR: 34-47), 47 years in San Diego (IQR: 38-55) and 42 years in Vancouver (IQR: 31-53). Those reporting recently assisting IDU initiation in Vancouver were, on average, younger (32 years, IQR: 23-40), which is potentially explained by the inclusion of young adults (aged 14-26 years) in the VDUS cohort. Recent IDU prevalence ranged by site from $86 \%$ in Tijuana to $65 \%$ in San Diego. Of those reporting recently assisting IDU initiation, however, 93\% in Tijuana, 94\% in San Diego and 97\% in Vancouver reported recent IDU. While most participants reported never having assisted IDU initiation prior to the past 6 months, approximately half of those who provided recent assistance had a history of doing so.

While a minority of participants reported recent housing insecurity across site, a greater proportion of those who had recently assisted IDU initiation reported housing insecurity. Similarly, a greater proportion of those who had recently assisted IDU initiation reported recently being stopped by law enforcement as compared with those who had not. A minority of participants reported recent incarceration, ranging from $9 \%$ in Vancouver to $27 \%$ in Tijuana. Only $3 \%$ of participants in Tijuana reported recent enrolment in OAT, likely reflective of lack of access to available services in the region, although OAT enrolment was higher in both San Diego $(20 \%)$ and Vancouver (49\%). Of those reporting recently assisting IDU initiation, only 6\% in San Diego and 35\% in Vancouver reported recent OAT enrolment.

Homogeneity across sites in reporting recently providing IDU initiation assistance

Compared with reporting recent IDU, reporting no recent IDU was associated with at least a $36 \%$ reduced likelihood of having recently assisted IDU initiation across all three sites (pRR: 0.21, 95\% CI: 0.07 to 0.64 , see table 2). Similarly, strong evidence across all three sites indicated that having a history of assisting IDU initiation increased the likelihood of recently assisting initiation by at least $249 \%$ (pRR: 4.83 , 95\% CI: 3.49 to 6.66 ). Identifying as male was associated with an increased likelihood of recently assisting initiation, although the point estimate of the pooled effect and the range of the CI (pRR: 1.29, $95 \%$ CI: 0.93 to 1.79 ) indicate that male gender may be associated with a $7 \%$ decrease up to a $79 \%$ increase in likelihood of recently assisting IDU initiation. Being recently stopped by law enforcement was associated with an $8 \%-107 \%$ increased likelihood of having recently assisted initiation across all three sites (pRR: 1.49, 95\% CI: 1.08 to 2.07). Evidence across sites indicated that recent methamphetamine IDU (pRR: 2.77, 95\% CI: 1.92 to 3.98 ) and recent speedball IDU (pRR: 2.11, 95\% CI: 1.35 to 3.31) were associated with a higher likelihood of having recently assisted initiation. Recent non-injection drug use (heroin, cocaine and/or methamphetamine) was associated with an increased likelihood of assisting IDU initiations, although the pooled effect and CI (pRR: 1.30, 95\% CI: 0.93 to 1.81 ) indicate that recent non-IDU may be associated with a $7 \%$ decrease up to an $81 \%$ increase in the likelihood of recently assisting IDU initiation.

\section{Heterogeneity across site in reporting recently providing IDU initiation assistance}

The association of age with recently assisting initiation was heterogenous across site $\left(\mathrm{I}^{2}=51.73 \%\right)$. Specifically, a 10 -year increase in age being associated with a decreased likelihood of recently assisting in both Tijuana (RR: 0.54, 95\% CI: 0.31 to 0.90 ) and Vancouver (RR: $0.66,95 \%$ CI: 0.43 to 0.90 ), while the direction of effect could not be confidently determined for San Diego (RR: 1.02, 95\% CI: 0.96 to 1.08). The association of years since first injection and recently assisting initiation was barely heterogenous across site $\left(I^{2}=0.20 \%\right)$, although the direction of effect could not be confidently determined for any of the three sites. The effect of housing insecurity was also heterogeneous across site $\left(\mathrm{I}^{2}=40.11 \%\right)$. In Tijuana, while recent housing insecurity was associated with between a $14 \%$ decrease and a $233 \%$ increase in likelihood (RR: 1.69, 95\% CI: 0.86 to 3.33 ), the existence and direction of effect could not be determined for San Diego (RR: 1.25, 95\% CI: 0.46 to 3.38 ) or Vancouver (RR: $0.84,95 \%$ CI: 0.56 to 1.29). Additionally, the association of recent incarceration and recently assisting initiation was heterogenous across site $\left(\mathrm{I}^{2}=56.99 \%\right)$, with evidence that the risk associated with recent incarceration on having recently assisted in 
Table 1 Recent injection initiation assistance provision and related factors among people who inject drugs in San Diego, USA; Tijuana, Mexico and Vancouver, Canada, 2014-2018 ( $n=2944)$

Tijuana San Diego

Did not recently Recently assisted Did not recently assist injection injection initiation assist injection

Variable initiation $(n=725)$

Age (mean (SD))

$40.72(9.13)$

$(n=41)$ initiation $(n=335)$

Gender

\begin{tabular}{|lcccccc|}
\hline Female & $289(39.9)$ & $12(29.3)$ & $94(28.1)$ & $4(22.2)$ & $657(37.9)$ & $31(35.6)$ \\
\hline Male & $436(60.1)$ & $29(70.7)$ & $234(70.3)$ & $14(77.8)$ & $1078(62.1)$ & $56(64.4)$ \\
\hline Transgender & $0(0.0)$ & $0(0.0)$ & $5(1.5)$ & $0(0.0)$ & -- & -- \\
\hline $\begin{array}{l}\text { Years since first } \\
\text { injection (mean }\end{array}$ & $19.68(9.97)$ & $19.23(8.85)$ & $23.98(13.19)$ & $23.06(12.53)$ & $21.05(13.50)$ & $12.06(10.60)$
\end{tabular}

$(\mathrm{SD}))$

Recent active IDU

$\begin{array}{ccccccc}\text { Yes } & 625(86.2) & 38(92.7) & 231(69.0) & 17(94.4) & 1133(65.2) & 85(96.6) \\ \text { No } & 100(13.8) & 3(7.3) & 104(31.0) & 1(5.6) & 604(34.8) & 3(3.4)\end{array}$

History of having assisted IDU initiation

$\begin{array}{lrrrrrr}\text { No } & 645(89.0) & 22(53.7) & 222(66.3) & 8(44.4) & 1399(80.5) & 35(39.8) \\ \text { Yes } & 80(11.0) & 19(46.3) & 113(33.7) & 10(55.6) & 338(19.5) & 53(60.2)\end{array}$

Recent housing insecurity

\begin{tabular}{|c|c|c|c|c|c|c|}
\hline No & $605(83.4)$ & 31 (75.6) & $250(74.6)$ & 12 (66.7) & $1287(74.1)$ & $51(58.0)$ \\
\hline Yes & $120(16.6)$ & $10(24.4)$ & 85 (25.4) & 6 (33.3) & 449 (25.9) & 37 (42.0) \\
\hline
\end{tabular}

Recently stopped by law enforcement

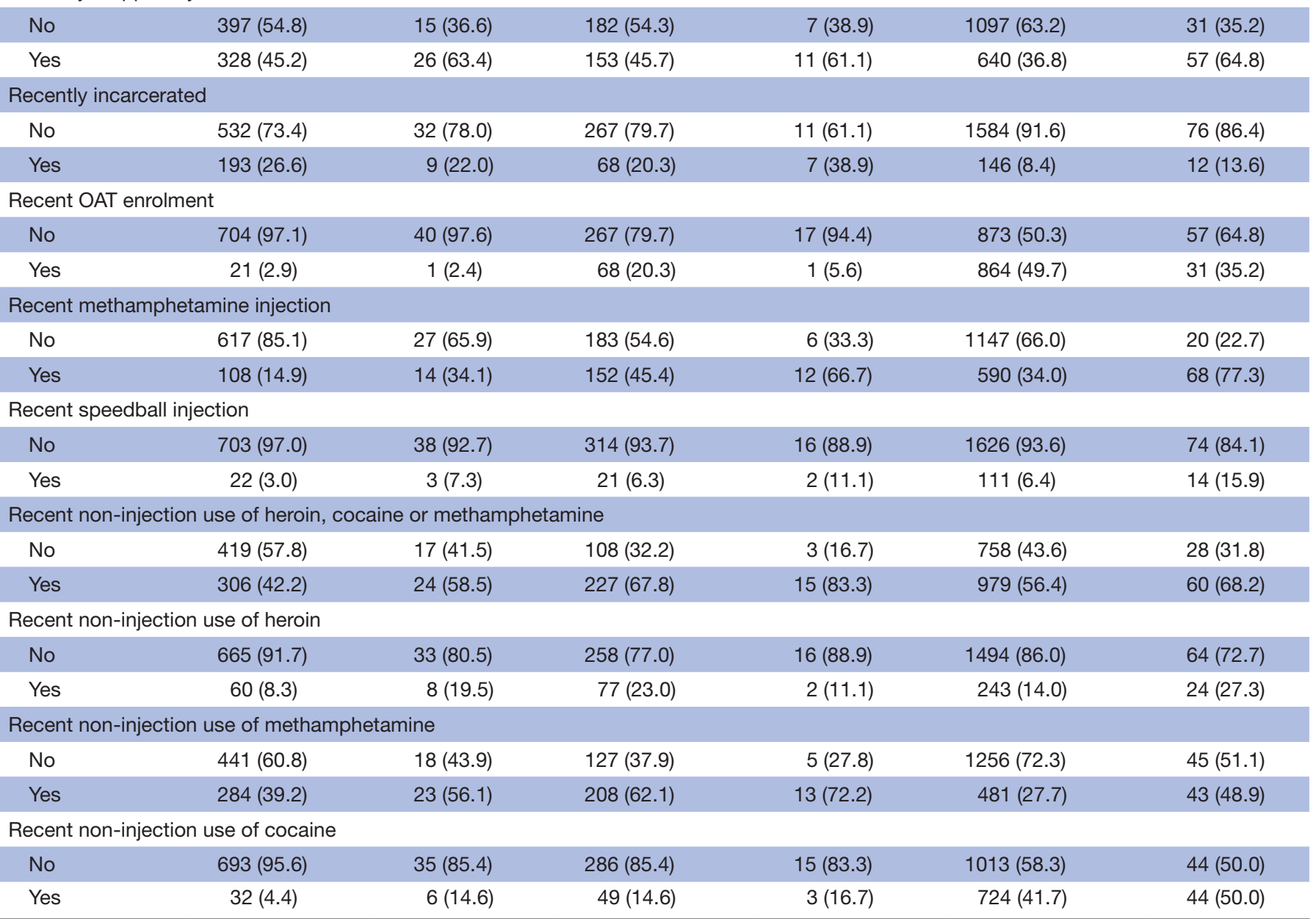


Table 1 Continued

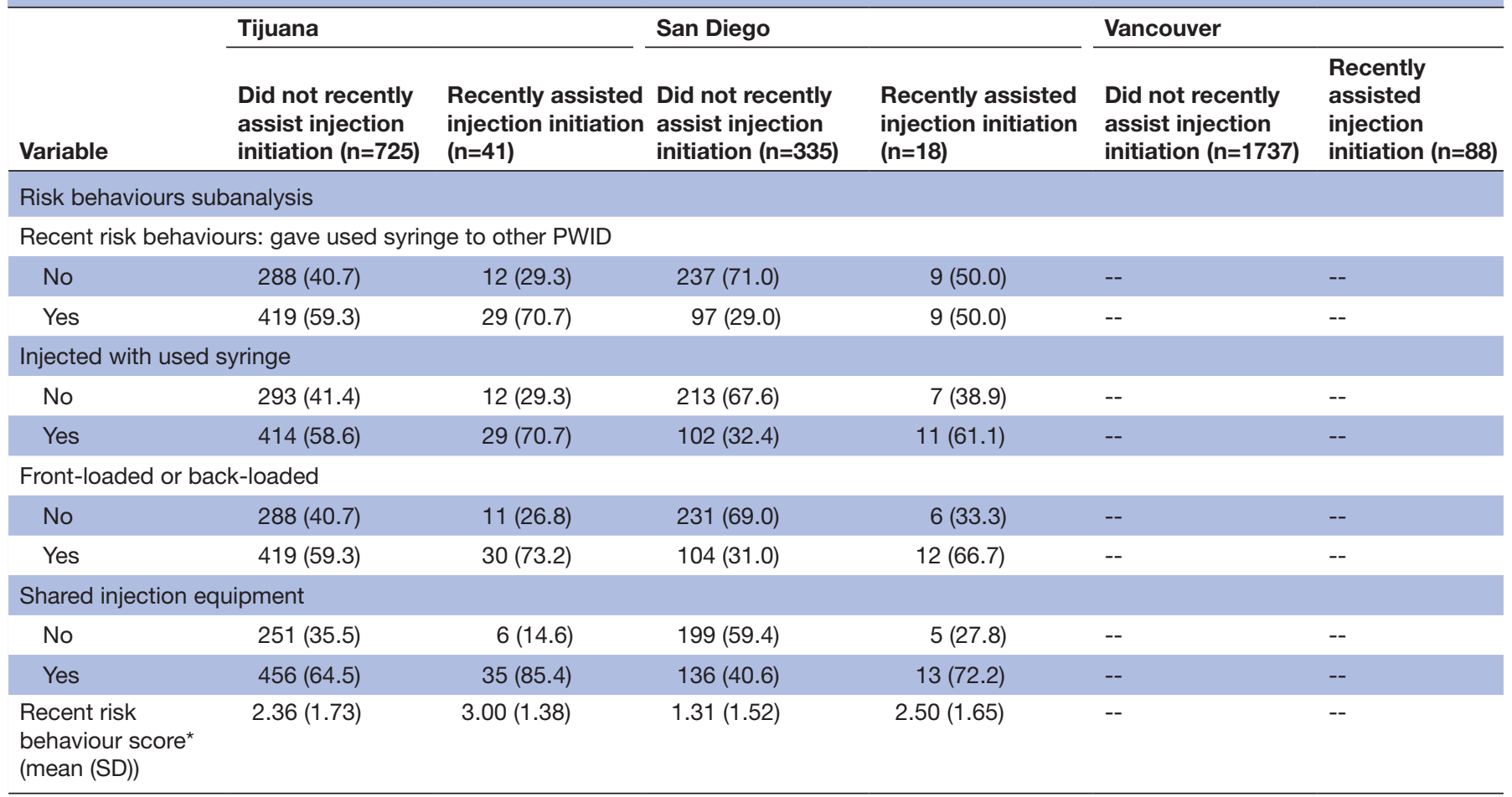

*Recent risk behaviour score is the sum of how many of the four individual risk behaviours participants responded recently having performed, resulting in a score between 0 and 4 .

IDU, injection drug use; OAT, opioid agonist treatment; PWID, people who inject drugs.;

San Diego could range from having no impact to a $478 \%$ increase in the likelihood of recently assisting IDU initiation (RR: $2.40,95 \%$ CI: 1.00 to 5.78 ). While the direction of the effect of recent IDU on assisting IDU initiation was consistent across site, we found the magnitude of effect to be heterogenous across site $\left(\mathrm{I}^{2}=50.40 \%\right)$. This is likely because not recently injecting had a weaker inverse association with assisting initiation in Tijuana (RR: 0.52) compared with San Diego (RR: 0.14) and Vancouver (RR: 0.11). The association of recent non-injection heroin use with recently assisting IDU initiation was heterogenous across site $\left(I^{2}=78.36 \%\right)$; in Tijuana, the increased likelihood of recently assisting initiation in Tijuana associated with this factor ranged from $28 \%$ to $381 \%$ (RR: $2.49,95 \%$ CI: 1.28 to 4.81 ).

\section{Risk behaviour subanalysis of San Diego and Tijuana}

The effect of recent IDU-related risk behaviours was assessed for the Tijuana and San Diego cohorts only (table 1). Across both sites, strong evidence indicated that recent piggybacking (sharing drugs via front-loading or back-loading) (pRR: $1.98,95 \%$ CI: 1.10 to 3.55 ) and recently sharing IDU preparation equipment (ie, cookers, cotton, water) (pRR: $2.44,95 \%$ CI: 1.18 to 5.02 ) were associated with an increased likelihood of recently assisting initiation. As above, a risk score, between 0 and 4 , was calculated for each participant by summing the number of risk behaviours participants indicated they had recently performed. Across both sites, an increased score was associated with a $23 \%-48 \%$ increased likelihood of recently assisting initiation (pRR: 1.23, 95\% CI: 1.02 to 1.48$)$.

\section{DISCUSSION}

This is the first study to pool findings across distinct cohort studies to assess factors associated with IDU initiation events. The findings presented indicate that, like other dynamic population-level health phenomena such as infectious disease epidemics, the dissemination of IDU initiation assistance across vulnerable populations is driven by both shared and setting-specific factors. For example, we found that a history of assisting IDU initiation, recently being stopped by law enforcement, and recent methamphetamine and speedball injection were associated with an increased likelihood of recently assisting IDU initiation across all three sites. These findings are consistent with prior literature. Evidence suggests that a minority of PWID are responsible for assisting the IDU initiation of a majority of injection-naïve individuals ${ }^{10}$ and having a history of assisting IDU initiation may be an effective proxy measurement for capturing individuals who assist many IDU initiations. In Tijuana, law enforcement has been found to focus on neighbourhoods with established and visible drug markets ${ }^{34}$ and, as such, it is possible that PWID who are most visible to law enforcement (such as those living in Vancouver's Downtown Eastside or 


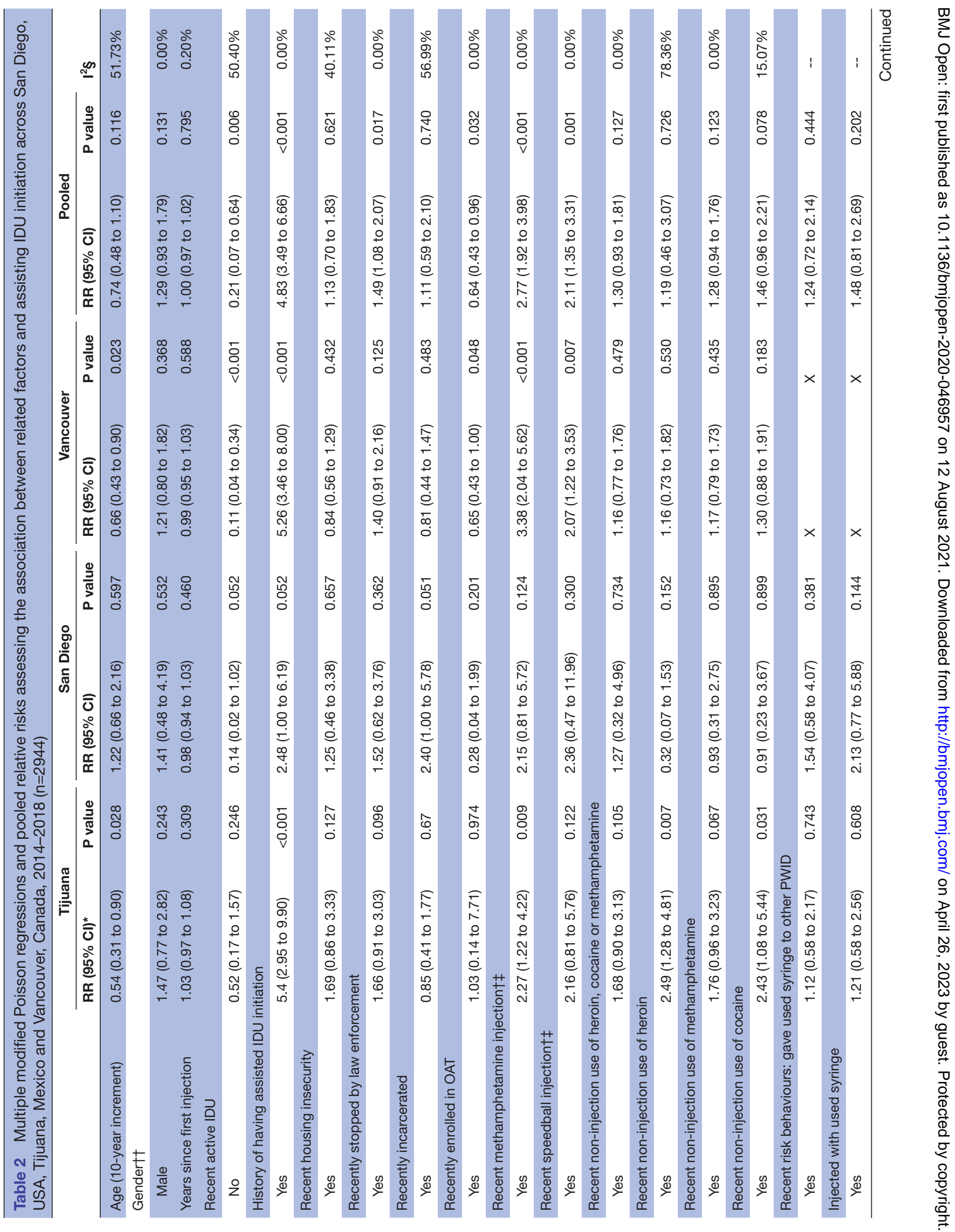




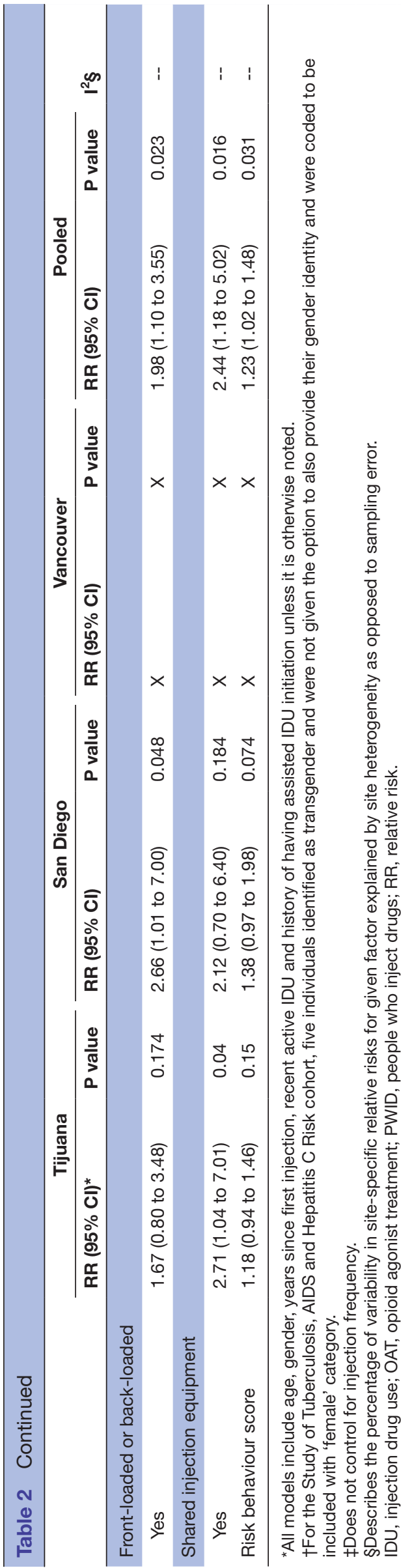

by the Tijuana River Canal) may also be most visible to injection-naïve individuals seeking initiation assistance. And, finally, while most of the research connecting stimulant use with increased likelihood of providing IDU initiation assistance used data from the same cohorts, ${ }^{11} 1621$ it appears that the use of crystal methamphetamine may play an important role for street youth who use drugs, including assisting them in staving off hunger, heightening sexual pleasure and making it easier to behave in a 'socially acceptable' manner, compared with other available drugs. ${ }^{35}$ Given that crystal methamphetamine use is associated with increased risk of initiating IDU, ${ }^{37}$ the utility of crystal methamphetamine use among street-involved youth makes this population particularly vulnerable to IDU initiation. Furthermore, we found that reporting not engaging in recent IDU and recent OAT enrolment were associated with a decreased likelihood of assisting IDU initiation across all three sites. These results have implications for efforts to prevent or delay transitions to IDU and other injection-related harms, in a range of sociocultural and geographic settings.

The current study highlights the considerable heterogeneity in the influence of factors on recently assisting IDU initiation-including recent non-injection heroin and cocaine use, recent incarceration and recent housing insecurity-which we found were influential in only specific settings. For example, recent non-injection heroin use was positively associated with recently assisting IDU initiation in Tijuana but, within the study sample in San Diego (a contiguous metropolitan setting), was negatively associated with recently assisting. This may be explained by a historically high geographic concentration of non-injection drug use and IDU in the Tijuana River canal, where up to 1000 people were recently believed to have resided, ${ }^{38}$ whereas San Diego does not have a location with a similarly dense concentration of PWID and people who use drugs (PWUD) through other routes of administration cohabitating. Thus, we may consider one source of site-specific heterogeneity as the presence of geographically concentrated public injecting such as that found both in the Tijuana River canal and Vancouver's Downtown Eastside neighbourhood, which has a disproportionately dense and stable population of PWID. ${ }^{38} 39$ Locations with higher concentrations of public IDU may facilitate interactions between PWID and injection-naive PWUD and thereby increase the likelihood of IDU initiation assistance occurring. ${ }^{40}{ }^{41}$ However, future research is required to further examine the factors that may explain site-specific outcomes. In the case of public IDU, we hypothesise that the more visible IDU is to injection-naive individuals, the more likely it is that observed PWID will be in positions to provide IDU initiation assistance.

This study implies directions for future research and interventions aimed at disrupting IDU transition events and related harms. Break the Cycle (BTC) and the adapted Change the Cycle, for example, are behavioural interventions aimed at reducing the likelihood that PWID assist IDU initiation in the future. ${ }^{42} 43$ The interventions 
use motivational interviewing, role playing and resource education to empower PWID to not assist IDU initiation. ${ }^{42}{ }^{43}$ Recent preliminary evidence from New York City, USA and Tallinn, Estonia ${ }^{43}$ as well as Toronto, Canada ${ }^{42}$ indicate potential efficacy of this programme. Our findings indicate that, across settings, engaging current PWID who inject methamphetamine and/or speedball, are not enrolled in OAT, and/or with a history of assisting IDU initiation may lead to improved efficacy of BTC. Furthermore, they indicate that factors such as incarceration and housing insecurity should be incorporated into sampling strategies on a context-specific basis.

With respect to other interventional approaches, we found that recent OAT enrolment is associated with a decreased likelihood of assisting IDU initiation, even though we had insufficient power to assess the direction and magnitude of this relationship in Tijuana (as only $3 \%$ of our Tijuana sample reported recent OAT enrolment). This is likely explained in large part by the lack of available OAT treatment in Tijuana, the prohibitive cost of OAT, ${ }^{44}$ stigma related to $\mathrm{OAT}^{38}$ as well as restrictions on OAT provision, ${ }^{45}$ and suggests a critical limitation of the use of OAT in under-resourced settings as a mode to reduce IDU initiation assistance among PWID. This is in juxtaposition to US and Canadian settings, where policies promoting a highly regulated expansion of OAT services in response to the current opioid crisis continue to expand. ${ }^{46}$ It is noteworthy, though, that OAT regulatory environments in the USS (which are stricter and controlled by federal policy) and Canada (which are more relaxed and subject to provincial policy), ${ }^{46}$ create unique contexts which may influence the efficacy and collateral health effects of OAT on the incidence of IDU initiation assistance provision. It is clear that future research that seeks to assess the potential use of OAT as an IDU initiation prevention intervention in other contexts must account for settingspecific policies surrounding the provision of substance use treatment. ${ }^{47}$

\section{LIMITATIONS}

This study has limitations inherent to exploratory analysis of observational data from multiple distinct cohort studies. For instance, our ability to assess heterogeneity between sites was limited by two factors: limited statistical power to analyse site-specific data (particularly for Tijuana and San Diego); and the limited number of sites $(n=3)$ from which we pooled data. These findings must therefore be interpreted in light of this uncertainty. In particular, because of the low power that we have to detect heterogeneity we can be confident in the presence of heterogeneity among those variables identified as such given the magnitude of heterogeneity required to generate signal. We also caution that an $\mathrm{I}^{2}$ of $0 \%$ for a given factor does not mean that that factor is homogenous across sites, but, more likely, that we were not powered to detect heterogeneity.
In addition, this study has limitations that are typical of observational cross-sectional research. For example, nonprobability sampling was employed, and thus the participants sampled may not be representative of the broader PWID population in each study setting. In particular, we likely were able to recruit the most visible PWID and PWID most connected with health and social services. As such, it is possible that our sample under-represented two vulnerable groups of PWID: (1) those who inject alone and (2) those without access to medical and social services. The population under study is also highly mobile and the high degree of human traffic between San Diego and Tijuana opens up the possibility that some of the IDU initiation assistance events reported by San Diego participants may have occurred in Tijuana, and vice versa. Furthermore, providing IDU initiation assistance is highly stigmatised and sensitive in nature. ${ }^{48}$ As a result, relying on self-report within the current study likely led to under-reporting of this behaviour. If patterns of underreporting are not explained by the factors explored in this study, this bias would likely skew results towards the null. We highlight that our findings are consistent with research that a minority of PWID are responsible for providing assistance to the IDU initiation of a majority of PWID, ${ }^{10}$ and, as such, we note that future research and interventions may want to focus specifically on this subpopulation. Further research is needed to determine if under-reporting is explained by any of these factors. Finally, data for this study were collected at the onset of the emergence of fentanyl in the illicit drug market. ${ }^{49}$ It is likely that concerns over drug contamination have resulted in modifications in IDU practices and, therefore, will be important for future studies to investigate how the emergence of fentanyl has impacted the provision of IDU initiation assistance. It is possible that the presence of fentanyl will increase the perceived importance of receiving IDU initiation assistance from experienced PWID, as injection-naïve individuals may rely on these experienced individuals as a safety precaution.

\section{CONCLUSION}

The current study is the first to pool cross-national data to assess commonality and heterogeneity in factors influencing IDU initiation assistance provision across distinct settings. These findings can inform interventions and policies seeking to prevent IDU initiation across distinct sociocultural contexts. Furthermore, our results imply that interventions targeting transitions into IDU and injection-related harms will need to move past a 'onesize-fits-all' approach and be adapted to address unique factors specific to each geographic and sociocultural context.

\section{Author affiliations}

${ }^{1}$ Department of Medicine, University of California San Diego, La Jolla, California, USA

${ }^{2}$ Interdisciplinary Research on Substance Use Joint Doctoral Program, San Diego State University and University of California San Diego, San Diego, California, USA 
${ }^{3}$ School of Social Work, San Diego State University, San Diego, California, USA ${ }^{4}$ Biostatistics Research Center, Herbert Wertheim School of Public Health and Human Longevity Science, University of California San Diego, La Jolla, California, USA

${ }^{5}$ Health Sciences, Simon Fraser University, Burnaby, British Columbia, Canada ${ }^{6}$ British Columbia Centre on Substance Use, Vancouver, British Columbia, Canada ${ }^{7}$ Family Medicine and Public Health, University of California San Diego, La Jolla, California, USA

${ }^{8}$ Department of Medicine, University of British Columbia, Vancouver, British Columbia, Canada

${ }^{9}$ School of Public Policy, Simon Fraser University, Vancouver, British Columbia, Canada

${ }^{10}$ Department of Public Health, CSU Fullerton, Fullerton, California, USA

${ }^{11}$ Centre on Drug Policy Evaluation, Unity Health Toronto, Toronto, Ontario, Canada

Contributors CM contributed to project conceptualisation, analytic design, data analysis and original manuscript writing. SAM contributed to project conceptualisation, analytic design and manuscript editing. SJ, XS and KC contributed to analytic design and manuscript editing. KH, PG-Z, SAS, RSG, MJM and $\mathrm{KDeB}$ contributed to data collection and manuscript editing. DW contributed to project conceptualisation, project supervision, funding acquisition, data collection and manuscript editing.

Funding PRIMER and Dan Werb are supported by a US National Institute on Drug Abuse Avenir Award (DP2- DA040256-01), the Canadian Institutes of Health Research via a New Investigator Award, the Ontario Ministry of Research, Innovation and Science via an Early Researcher Award and by a project grant from the Canadian Institutes of Health Research. El Cuete IV was supported through NIDA grant R37 DA019829. STAHR II was supported through NIDA grant R01DA031074. VDUS was supported by NIDA grant U01DA038886 and the ACCESS Study was supported by NIDA grant U01DA021525.

Competing interests None declared.

Patient and public involvement Patients and/or the public were not involved in the design, or conduct, or reporting, or dissemination plans of this research.

Patient consent for publication Not required.

Ethics approval The PRIMER study was granted ethical approval by the University of California San Diego (IRB 150866). Each study site received ethical approval from their respective institutional review boards. All participants provided consent to participate prior to enrolment in their respective cohort.

Provenance and peer review Not commissioned; externally peer reviewed.

Data availability statement No data are available. The data for this study cannot be made public due to human subjects' protections.

Open access This is an open access article distributed in accordance with the Creative Commons Attribution Non Commercial (CC BY-NC 4.0) license, which permits others to distribute, remix, adapt, build upon this work non-commercially, and license their derivative works on different terms, provided the original work is properly cited, appropriate credit is given, any changes made indicated, and the use is non-commercial. See: http://creativecommons.org/licenses/by-nc/4.0/.

ORCID iD

Dan Werb http://orcid.org/0000-0003-0614-9386

\section{REFERENCES}

1 United States Department of Health and Human Services. What is the U.S. overdose crisis? 2019. Available: https://www.hhs.gov/ opioids/about-the-epidemic/index.html

2 National Institute on Drug Abuse. Opioid overdose crisis, 2019. Available: https://www.drugabuse.gov/drugs-abuse/opioids/opioidoverdose-crisis [Accessed 12 Mar 2019].

3 Haskins J. Suicide, opioids tied to ongoing fall in US life expectancy: third year of drop. Nation \& Heal 2019;49.

4 Degenhardt L, Peacock A, Colledge S, et al. Global prevalence of injecting drug use and sociodemographic characteristics and prevalence of HIV, HBV, and HCV in people who inject drugs: a multistage systematic review. Lancet Glob Health 2017;5:e1192-207.

5 Colledge S, Peacock A, Leung J, et al. The prevalence of non-fatal overdose among people who inject drugs: a multi-stage systematic review and meta-analysis. Int J Drug Policy 2019;73:172-84.
6 Goldsamt LA, Harocopos A, Kobrak P, et al. Circumstances, pedagogy and rationales for injection initiation among new drug injectors. J Community Health 2010;35:258-67.

7 Garfein RS, Doherty MC, Monterroso ER, et al. Prevalence and incidence of hepatitis $C$ virus infection among young adult injection drug users. J Acquir Immune Defic Syndr Hum Retrovirol 1998;18 Suppl 1:S11-9.

8 Werb D, Buxton J, Shoveller J, et al. Interventions to prevent the initiation of injection drug use: a systematic review. Drug Alcohol Depend 2013;133:669-76.

9 Vlahov D, Fuller CM, Ompad DC, et al. Updating the infection risk reduction hierarchy: preventing transition into injection. J Urban Health 2004;81:14-19.

10 Gicquelais RE, Werb D, Marks C, et al. Prevalence and correlates of providing and receiving assistance with the transition to injection drug use. Epidemiol Rev 2020;42:4-18.

11 Meyers SA, Scheim A, Jain S, et al. Gender differences in the provision of injection initiation assistance: a comparison of three North American settings. Harm Reduct J 2018;15:59.

12 Ben Hamida A, Rafful C, Jain S, et al. Non-injection drug use and injection initiation assistance among people who inject drugs in Tijuana, Mexico. J Urban Health 2018;95:83-90.

13 Melo JS, Garfein RS, Hayashi K, et al. Do law enforcement interactions reduce the initiation of injection drug use? an investigation in three North American settings. Drug Alcohol Depend 2018;182:67-73.

14 Bouck Z, Jain S, Sun X, et al. Recent incarceration and risk of firsttime injection initiation assistance: a prospective cohort study of persons who inject drugs. Drug Alcohol Depend 2020;212:107983.

15 Marks C, Bouck Z, Jain S, et al. The impact of recent homelessness on the provision of injection drug use initiation assistance among persons who inject drugs in Tijuana, Mexico and Vancouver, Canada. Drug Alcohol Depend 2021;225:108829.

16 Mittal ML, Jain S, Sun S, et al. Opioid agonist treatment and the process of injection drug use initiation. Drug Alcohol Depend 2019;197:354-60.

17 Mittal ML, Vashishtha D, Sun S, et al. History of medication-assisted treatment and its association with initiating others into injection drug use in San Diego, Ca. Subst Abuse Treat Prev Policy 2017;12:42.

18 Marks C, Borquez A, Jain S, et al. Opioid agonist treatment scale-up and the initiation of injection drug use: a dynamic modeling analysis. PLoS Med 2019;16:e1002973.

19 Werb D, Garfein R, Kerr T, et al. A socio-structural approach to preventing injection drug use initiation: rationale for the primer study. Harm Reduct J 2016;13:25.

20 Robertson AM, Garfein RS, Wagner KD, et al. Evaluating the impact of Mexico's drug policy reforms on people who inject drugs in Tijuana, B.C., Mexico, and San Diego, Ca, United States: a binationa mixed methods research agenda. Harm Reduct $J$ 2014;11:4.

21 Rafful C, Melo J, Medina-Mora ME, et al. Cross-border migration and initiation of others into drug injecting in Tijuana, Mexico. Drug Alcohol Rev 2018;37 Suppl 1:S277-84.

22 Shannon K, Kerr T, Strathdee SA, et al. Prevalence and structural correlates of gender based violence among a prospective cohort of female sex workers. BMJ 2009;339:b2939.

23 Zou G. A modified poisson regression approach to prospective studies with binary data. Am J Epidemiol 2004;159:702-6.

24 Tan J, Altice FL, Madden LM, et al. Effect of expanding opioid agonist therapies on the HIV epidemic and mortality in Ukraine: a modelling study. Lancet HIV 2020;7:e121-e128.

$25 \mathrm{Knol}$ MJ, Le Cessie S, Algra A, et al. Overestimation of risk ratios by odds ratios in trials and cohort studies: alternatives to logistic regression. CMAJ 2012;184:895-9.

26 Blettner M, Sauerbrei W, Schlehofer B, et al. Traditional reviews, meta-analyses and pooled analyses in epidemiology. Int J Epidemiol 1999;28:1-9.

27 Higgins JPT, Thompson SG. Quantifying heterogeneity in a metaanalysis. Stat Med 2002;21:1539-58.

28 Viechtbauer W. Conducting meta-analyses in $\mathrm{R}$ with the metafor package. J Stat Softw 2010;36

29 Rafful C, Jain S, Sun X, et al. Identification of a Syndemic of bloodborne disease transmission and injection drug use initiation at the US-Mexico border. J Acquir Immune Defic Syndr 2018;79:559-65.

30 Wasserstein RL, Schirm AL, Lazar NA. Moving to a World Beyond " $p$ $<0.05$ ". Am Stat 2019:73:1-19.

31 Hurlbert SH, Levine RA, Utts J. Coup de Grâce for a Tough Old Bull: "Statistically Significant" Expires. Am Stat 2019;73:352-7.

32 Amrhein V, Greenland S, McShane B. Scientists rise up against statistical significance. Nature 2019;567:305-7.

33 Cummins KM, Marks C. Farewell to Bright-Line: a guide to reporting quantitative results without the S-Word. Front Psychol 2020;11:815. 
34 Gaines T, Werb D, Arredondo J, et al. The spatial-temporal relationship of policing in areas with high drug activity. Drug A/cohol Depend 2015;156:e77.

35 Werb D, Kerr T, Zhang R, et al. Methamphetamine use and malnutrition among street-involved youth. Harm Reduct $J$ 2010;7:5.

36 Fast D, Kerr T, Wood E, et al. The multiple truths about crystal Meth among young people entrenched in an urban drug scene: a longitudinal ethnographic investigation. Soc Sci Med 2014;110:41-8.

37 Werb D, Kerr T, Buxton J, et al. Crystal methamphetamine and initiation of injection drug use among street-involved youth in a Canadian setting. Can Med Assoc J 2013;185:1569-75.

38 Rafful C, Medina-Mora ME, González-Zúñiga P, et al. "Somebody Is Gonna Be Hurt": Involuntary Drug Treatment in Mexico. Med Anthropol 2020;39:139-52.

39 Chami G, Werb D, Feng C, et al. Neighborhood of residence and risk of initiation into injection drug use among street-involved youth in a Canadian setting. Drug Alcohol Depend 2013;132:486-90.

40 Tobin KE, Davey-Rothwell M, Latkin CA. Social-level correlates of shooting gallery attendance: a focus on networks and norms. AIDS Behav 2010;14:1142-8.

41 Mazhnaya A, Tobin KE, Owczarzak J. Association between injection in public places and HIV/HCV risk behavior among people who use drugs in Ukraine. Drug Alcohol Depend 2018;189:125-30.
42 Strike C, Rotondi M, Kolla G, et al. Interrupting the social processes linked with initiation of injection drug use: results from a pilot study. Drug Alcohol Depend 2014;137:48-54.

43 Des Jarlais D, Uuskula A, Talu A, et al. Implementing an Updated "Break the Cycle" Intervention to Reduce Initiating Persons into Injecting Drug Use in an Eastern European and a US "opioid epidemic" Setting. AIDS Behav 2019;23:2304-14.

44 Burgos JL, Cepeda JA, Kahn JG, et al. Cost of provision of opioid substitution therapy provision in Tijuana, Mexico. Harm Reduct $J$ 2018;15:28

45 Goodman-Meza D, Medina-Mora ME, Magis-Rodríguez C, et al. Where is the opioid use epidemic in Mexico? A cautionary tale for policymakers South of the US-Mexico border. Am J Public Health 2019;109:73-82.

46 Priest KC, Gorfinkel L, Klimas J, et al. Comparing Canadian and United States opioid agonist therapy policies. Int J Drug Policy 2019;74:257-65.

47 Vashishtha D, Mittal ML, Werb D. The North American opioid epidemic: current challenges and a call for treatment as prevention. Harm Reduct J 2017;14:7.

48 Guise A, Horyniak D, Melo J, et al. The experience of initiating injection drug use and its social context: a qualitative systematic review and thematic synthesis. Addiction 2017;112:2098-111.

49 Ciccarone D. The triple wave epidemic: supply and demand drivers of the US opioid overdose crisis. Int J Drug Policy 2019;71:183-8. 